\title{
Light propagation in layered photonic crystals with admixture layers
}

\author{
Rumyantsev V.V., Fedorov S.A. and Gumennyk K.V. \\ A. A. Galkin Donetsk Physico-Technical Institute of NASU, \\ 72 R. Luxembourg St., 83114 Donetsk, Ukraine. \\ E-mail: rumyants@teor.fti.ac.donetsk.ua
}

Received: 29.01.2008

\begin{abstract}
The virtual-crystal approximation is used for numerical simulation of a polariton spectrum transformation in composite materials, consisting of alternating silicon and liquid crystal layers and randomly included admixture layers. The character of dependence of the bandgap width and the refractive index upon the concentration of admixture layers is discussed. It is shown that the energy structure of this imperfect superlattice can be significantly altered by implantation of appropriate defect layers.
\end{abstract}

Keywords: photonic crystal, $\mathrm{Si}$ - liquid crystal system, admixture layer, bandgap width.

PACS: 78.66.-W

UDC: 535.3

\section{Introduction}

Propagation of electromagnetic waves in thin films and layered crystalline media is currently drawing a close attention of researchers. The works [1-4] give the account of the related researches carried out for photonic crystals and [5] - for some composite materials based on silicon and liquid crystals. On the one hand, the interest towards these objects is due to their significance for electronics. On the other hand, this is because a notable advance of technology that allows growing of ultra-thin films and periodic structures with controlled characteristics.

There have been numerous theoretical and experimental studies (see, e.g., [6] and references therein) on exciton-like excitations in ideal dielectric superlattices. A general theory of optical waves in anisotropic crystals, including those formed of macroscopic layers, has been discussed in Ref. [7]. The authors [8] have investigated dispersion of polaritons in a superlattice with two admixture layers. At the same time, a considerable interest has been focused on non-ideal superlattices with an arbitrary number of admixture layers, as well as on dependence of polariton spectrum on the concentration of corresponding defects. Further development of the theory of layered structures evidently requires considering more complex models like superlattices with randomly included admixture layers of variable composition. A better understanding of how the optical proper-

Ukr. J. Phys. Opt. 2008, V9, №2 
ties of such the systems depend on the concentration of admixture layers would give a basis for modelling and constructing the layered materials with predetermined characteristics.

The method applied for calculating the polariton excitation spectra is rather similar to those used in cases of other quasi-particle excitations, like electronic, phononic etc. In the present work we employ the virtual-crystal approximation (VCA) [9, 10], based on configurational averaging, for description of polariton excitations in a macroscopically inhomogeneous medium. In general, it is a well-known method, though its utilization has been limited up to now to microscopic calculations of the quasiparticle excitation spectra in disordered systems [11]. Nonetheless, mathematical posing of the problem is similar in these two cases.

The VCA proposed originally by L. Nordheim and R. H. Parmenter [9] consists in replacing the exact one-electron potential (appropriate to a given configuration of atoms of the alloy) by its average taken over all of possible random configurations. This approximation is widely used in the studies for different disordered structures. For example, based on the pseudopotential scheme under the VCA, in which the effect of compositional disorder is involved, the authors [12] have studied the dependence of optoelectronic properties of $\mathrm{GaAs}_{x} \mathrm{Sb}_{1-x}$ on the alloy composition $x$. Within this approximation configurationally dependent parameters of Hamiltonian are replaced with their configurationally averaged values. Description of transformation of polariton spectrum in a sufficiently simple superlattice with the aid of VCA is the first step towards the study of imperfect systems. However, investigation of properties of the polariton spectra and the related physical quantities (density of elementary excitation states, characteristics of normal electromagnetic waves etc.) of less simple systems requires application of more complex methods. These are the method of coherent (one- or many-site) potential [11], the averaged T-matrix method [13] and their numerous modifications used for various particular problems.

In this work we model superlattices as a set of macroscopically homogeneous layers with randomly included extrinsic (with respect to the ideal superlattice) layers. The corresponding configuration-dependent material tensors in our model of imperfect superlattice are represented in terms of random quantities. After configurational averaging the translational symmetry of the system under consideration is "restored", thus allowing us to obtain the system of equations that defines the normal modes of electromagnetic waves propagating in one-dimensional "periodic" medium. Within the VCA we study the peculiarities of dependence of the bandgap width and the refractive index upon the concentration of admixture layers for the non-ideal system ' $\mathrm{Si}$ - liquid crystal'.

\section{Propagation of electromagnetic waves in inhomogeneous structures}

The dielectric $(\hat{\varepsilon}(\vec{r}))$ and magnetic $(\hat{\mu}(\vec{r}))$ permeabilities, which determine optical characteristics of a periodic medium, should satisfy periodic boundary conditions: 


$$
\hat{\varepsilon}(x, y, z)=\hat{\varepsilon}(x, y, z+d) \quad \hat{\mu}(x, y, z)=\hat{\mu}(x, y, z+d),
$$

where $d=\sum_{j=1}^{\sigma} a_{j}$ is the period of our superlattice, $\sigma$ the number of layers per elementary cell and $a_{j}$ thicknesses of the layers that form one-dimensional chain of elements oriented along $z$ axis. The material tensors $\hat{\varepsilon}$ and $\hat{\mu}$ of a crystalline superlattice with an arbitrary number of layers $\sigma$ have the following form in the coordinate representation:

$$
\left(\begin{array}{l}
\hat{\varepsilon}(z) \\
\hat{\mu}(z)
\end{array}\right)=\sum_{n, \alpha}\left(\begin{array}{l}
\hat{\varepsilon}_{n \alpha} \\
\hat{\mu}_{n \alpha}
\end{array}\right)\left\{\theta\left[z-(n-1) d-\left(\sum_{j=1}^{\alpha} a_{n j}-a_{n \alpha}\right)\right]-\theta\left[z-(n-1) d-\sum_{j=1}^{\alpha} a_{n j}\right]\right\}
$$

In Eq. (2) $\theta(z)$ denotes the Heaviside function, $n= \pm 1, \pm 2, \ldots$ the number of onedimensional crystal cells and the index $\alpha=1,2, \ldots, \sigma$ labels elements of the cell. Below we consider an imperfect system, in which disordering is linked with variation of composition of the admixture layers rather than their thickness, so that $a_{n \alpha} \equiv a_{\alpha}$. Within our model, configurationally dependent tensors $\hat{\varepsilon}_{n \alpha}, \hat{\mu}_{n \alpha}$ are expressed through the random quantities $\eta_{n \alpha}^{v}\left(\eta_{n \alpha}^{v}=1\right.$ if the $v(\alpha)$ th sort of layer is in the $(n \alpha)$ th site of crystalline chain, otherwise we have $\eta_{n \alpha}^{v}=0$ ):

$$
\left(\begin{array}{c}
\hat{\varepsilon}_{n \alpha} \\
\hat{\mu}_{n \alpha}
\end{array}\right)=\sum_{v(\alpha)}\left(\begin{array}{c}
\hat{\varepsilon}_{\alpha}^{v(\alpha)} \\
\hat{\mu}_{\alpha}^{v(\alpha)}
\end{array}\right) \eta_{n \alpha}^{v(\alpha)}
$$

Similarly to the solid quasi-particle approach, calculation of the polariton spectrum for the imperfect superlattice within the VCA is implemented through the replacement $\hat{\varepsilon} \rightarrow\langle\hat{\varepsilon}\rangle, \hat{\mu} \rightarrow\langle\hat{\mu}\rangle$, where angular parentheses mean a procedure of configurational averaging. In addition, from Eq. (3) and Ref. [9] we have the relation

$$
\left(\begin{array}{c}
\left\langle\hat{\varepsilon}_{n \alpha}\right\rangle \\
\left\langle\hat{\mu}_{n \alpha}\right\rangle
\end{array}\right)=\sum_{\alpha, v(\alpha)}\left(\begin{array}{c}
\hat{\varepsilon}_{\alpha}^{v(\alpha)} \\
\hat{\mu}_{\alpha}^{v(\alpha)}
\end{array}\right) C_{\alpha}^{v(\alpha)}
$$

where $C_{\alpha}^{v(\alpha)}$ is the concentration of $v(\alpha)$ th sort of admixture layer in the $\alpha$ th sublattice. Here a simple normalization condition $\sum_{v(\alpha)} C_{\alpha}^{v(\alpha)}=1$ holds true. It follows from Eq. (2) that the Fourier-amplitudes $\hat{\varepsilon}_{l}, \hat{\mu}_{l}$ and the averaged dielectric $\left(\left\langle\hat{\varepsilon}_{n \alpha}\right\rangle\right)$ and magnetic $\left(\left\langle\hat{\mu}_{n \alpha}\right\rangle\right)$ permeabilities of layers given by Eqs. (4) are related as

$$
\left(\begin{array}{c}
\hat{\varepsilon}_{l} \\
\hat{\mu}_{l}
\end{array}\right)=-\frac{i}{2 \pi l} \sum_{\alpha}\left(\begin{array}{l}
\left\langle\hat{\varepsilon}_{n \alpha}\right\rangle \\
\left\langle\hat{\mu}_{n \alpha}\right\rangle
\end{array}\right)\left\{\exp \left(i \frac{2 \pi}{d} l \sum_{j=1}^{\alpha} a_{j}\right)-\exp \left[i \frac{2 \pi}{d} l\left(\sum_{j=1}^{\alpha} a_{j}-a_{\alpha}\right)\right]\right\} .
$$


Since the configurational averaging "restores" translational symmetry of a crystalline system, the "acquired" translational invariance of our one-dimensional chain in the considered case of imperfect superlattice allows us to represent Maxwell equations in the form

$$
\nabla \times \vec{E}(\vec{r}, \omega)=\frac{i \omega}{c}\langle\hat{\mu}(z)\rangle \cdot \vec{H}(\vec{r}, \omega), \nabla \times \vec{H}(\vec{r}, \omega)=-\frac{i \omega}{c}\langle\hat{\varepsilon}(z)\rangle \cdot \vec{E}(\vec{r}, \omega),
$$

where harmonic time dependences of the electric and magnetic fields $\vec{E}(\vec{r}, \omega), \vec{H}(\vec{r}, \omega)$ are assumed.

According to the Floquet theorem, Fourier amplitudes $\vec{f}_{K, p}^{(E, H)}$ of the electric and magnetic fields satisfy the following relation:

$$
\left[\vec{\beta}+\left(K+p \frac{2 \pi}{d}\right) \vec{e}_{z}\right] \times\left(\begin{array}{c}
\vec{f}_{K, p}^{(H)} \\
\vec{f}_{K, p}^{(E)}
\end{array}\right)=\frac{\omega}{c}\left[\begin{array}{c}
-\sum_{l} \hat{\varepsilon}_{l} \cdot \vec{f}_{K, p-l}^{(E)} \\
\sum_{l} \hat{\mu}_{l} \cdot \vec{f}_{K, p-l}^{(H)}
\end{array}\right] .
$$

Here $\vec{\beta}$ is an arbitrary planar (i.e., lying in the XOY plane) wave vector, $\vec{e}_{z}$ a unit vector along the $z$ axis and $\vec{K}=(0,0, K)$ the Bloch vector. The system (7) defines the normal modes of electromagnetic waves that propagate in the "periodic" medium under analysis.

For simplicity, below we restrict our study to the case of light propagation along the $z$ axis $(\vec{\beta}=0)$ in a nonmagnetic lattice (with $\hat{\mu}=\hat{I}$ being a unit matrix). Like in Ref. [5], we treat the liquid-crystal layers as optically uniaxial $\left(\varepsilon_{i j}=\varepsilon_{x x} \delta_{x i} \delta_{j x}+\varepsilon_{y y} \delta_{y i} \delta_{j y}+\varepsilon_{z z} \delta_{z i} \delta_{j z}\right)$. It is obvious that $z z$-components of the tensor $\hat{\varepsilon}$ will not appear in the final formulae for the case of $\vec{K} \| z$ and, moreover, we have $\varepsilon_{x x}=\varepsilon_{y y} \equiv \varepsilon$. The same as in Ref. [7], we assume further that $K$ is close to the value defined by the Bragg condition, i.e. $\left|K-\frac{2 \pi}{d}\right| \approx K$ and $\quad c^{2} K^{2} \approx \omega^{2} \varepsilon_{0}$. This case corresponds to a resonance of plane waves between the components $\vec{f}_{K, p}^{(E, H)}$ at $p=0,-1$ (these terms dominate in the system of Eqs. (7)). After eliminating the $\vec{f}^{(H)}$ variables, we rewrite Eqs. (7) with respect to $\vec{f}^{(E)}$ as follows:

$$
\left[\begin{array}{cc}
K^{2}-\frac{\omega^{2}}{c^{2}} \varepsilon^{(0)} & -\frac{\omega^{2} \varepsilon^{(1)}}{c^{2}} \\
-\frac{\omega^{2} \varepsilon^{(-1)}}{c^{2}} & \left(K-\frac{2 \pi}{d}\right)^{2}-\frac{\omega^{2}}{c^{2}} \varepsilon^{(0)}
\end{array}\right]\left(\begin{array}{l}
f_{K, 0}^{(E)} \\
f_{K,-1}^{(E)}
\end{array}\right)=0,
$$

where $\varepsilon_{l=0} \equiv \varepsilon^{(0)}, \varepsilon_{l= \pm 1} \equiv \varepsilon^{( \pm 1)}$. Putting determinant of the system of Eqs. (8) to be zero, 
we obtain the dispersion relations $\omega_{ \pm}=\omega(K)$. Two roots $\omega_{ \pm}$of this equation define the boundaries of the spectral band: the roots become complex at the frequencies $\omega_{-}(K)<\omega<\omega_{+}(K)$ (the bandgap) and the electromagnetic waves decay (the Bragg reflection). The frequencies $\omega<\omega_{-}, \omega>\omega_{+}$correspond to propagating waves.

\section{Results and discussion}

Let us confine ourselves to the case of propagation of electromagnetic radiation in a nonmagnetic superlattice with two constituent layers, a Si-layer and a liquid-crystal layer, per elementary cell. The concentration and the dielectric permeability of the basic material in the first and second sublattices are denoted respectively as $C_{1}^{(1)}, \varepsilon_{1}^{(1)}$ and $C_{2}^{(1)}, \varepsilon_{2}^{(1)}$, with $\varepsilon_{1}^{(1)}=11.7$ and $\varepsilon_{2}^{(1)}=5.5$. For the admixture these quantities are denoted as $C_{1}^{(2)}, \varepsilon_{1}^{(2)}$ and $C_{2}^{(2)}, \varepsilon_{2}^{(2)}$. Simple transformations with account for the property $\left|\varepsilon^{(-1)}\right|=\left|\varepsilon^{(1)}\right|$ lead to the following relations for the refractive index $n \equiv c K / \omega$ of the system under study:

$$
n_{ \pm}^{2}\left(C_{1}^{(2)}, C_{2}^{(2)}\right)=\varepsilon^{(0)}\left(C_{1}^{(2)}, C_{2}^{(2)}\right) \pm\left|\varepsilon^{(1)}\left(C_{1}^{(2)}, C_{2}^{(2)}\right)\right| \cong \varepsilon^{(0)}\left[1 \pm \frac{\Delta \omega_{1}\left(C_{1}^{(2)}, C_{2}^{(2)}\right)}{\omega}\right],
$$

where $\left(n_{+}^{2}-n_{-}^{2}\right) / 2 \varepsilon^{(0)} \cong \Delta \omega_{1} / \omega$ and $\Delta \omega_{1}=\left|\omega_{+}-\omega_{-}\right|$is the width of the lowest bandgap. It follows from Eq. (9) that the quantity $\Delta \omega_{1}$ is determined by the corresponding coefficient of the Fourier expansion given by Eq. (5), which in this case is $\left|\varepsilon^{(1)}\right|$. In Refs. [5, 7] it has been shown that the bandgaps of higher orders are also determined by the corresponding Fourier coefficients of the dielectric permeability:

$$
\begin{aligned}
& \varepsilon^{(0)}=\left(\varepsilon_{1}^{(1)} f_{1} a_{1}+\varepsilon_{2}^{(1)} f_{2} a_{2}\right) / d, \\
& \left|\varepsilon^{(1)}\right|=\frac{1}{\pi}\left|\varepsilon_{2}^{(1)} f_{2}-\varepsilon_{1}^{(1)} f_{1}\right| \sin \pi a_{1} / d .
\end{aligned}
$$

The functions $f_{1}\left(C_{1}^{(2)}, \frac{\varepsilon_{1}^{(2)}}{\varepsilon_{1}^{(1)}}\right)=1-C_{1}^{(2)}\left(1-\frac{\varepsilon_{1}^{(2)}}{\varepsilon_{1}^{(1)}}\right)$ and $f_{2}\left(C_{2}^{(2)}, \frac{\varepsilon_{2}^{(2)}}{\varepsilon_{2}^{(1)}}\right)=1-C_{2}^{(2)}\left(1-\frac{\varepsilon_{2}^{(2)}}{\varepsilon_{2}^{(1)}}\right) \mathrm{de}-$ pend on the concentration of admixture layers and their relative dielectric permeability.

Fig. 1 shows the concentration dependence of the refractive index $n_{ \pm} \equiv c K / \omega_{ \pm}$ of the composite superlattice. It is readily seen that the form of the corresponding surfaces has a non-monotone character if the dielectric permeability of both admixtures is $\varepsilon_{i}^{(2)} / \varepsilon_{i}^{(1)} \ll 1(i=1,2)$ (the case (a)) or $\varepsilon_{i}^{(2)} / \varepsilon_{i}^{(1)} \gg 1(i=1,2)$. The dependences of $n_{+}$and $n_{-}$on $C_{1}^{(2)}$ and $C_{2}^{(2)}$ become monotonous in the case (b). The latter fact determines the behaviour of the lowest bandgap.

Ukr. J. Phys. Opt. 2008, V9, №2 


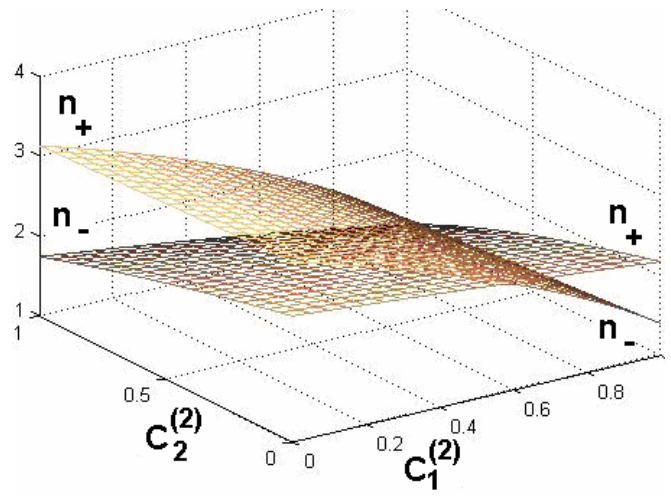

a

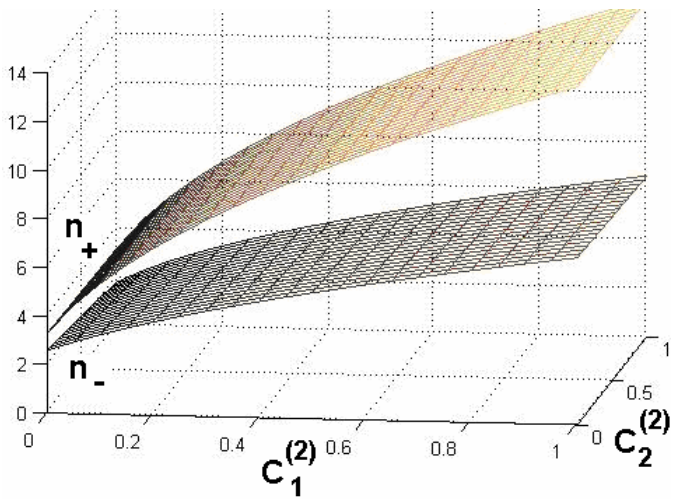

b

Fig. 1. Refractive index $n_{ \pm} \equiv c K / \omega_{ \pm}$of the composite superlattice comprising alternating silicon and liquid-crystal layers vs. concentrations of the admixture layers (under the condition $a_{1} / a_{2}=1$ ): (a) $\varepsilon_{1}^{(2)} / \varepsilon_{1}^{(1)}=0.1$ and $\varepsilon_{2}^{(2)} / \varepsilon_{2}^{(1)}=0.2 ;$ (b) $\varepsilon_{1}^{(2)} / \varepsilon_{1}^{(1)}=20$ and $\varepsilon_{2}^{(2)} / \varepsilon_{2}^{(1)}=0.2$.

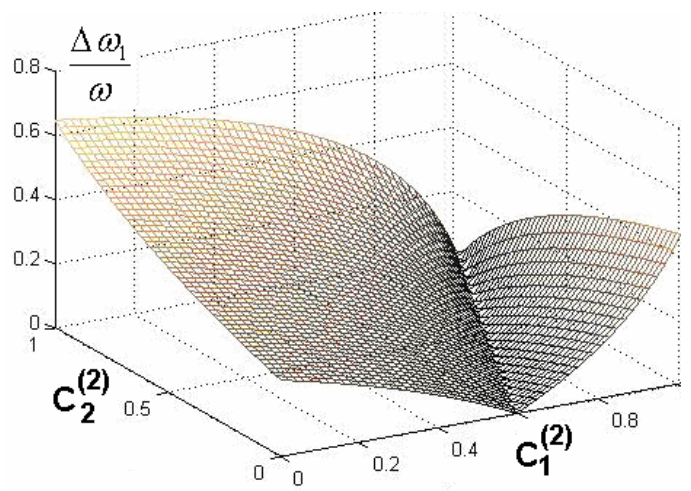

a

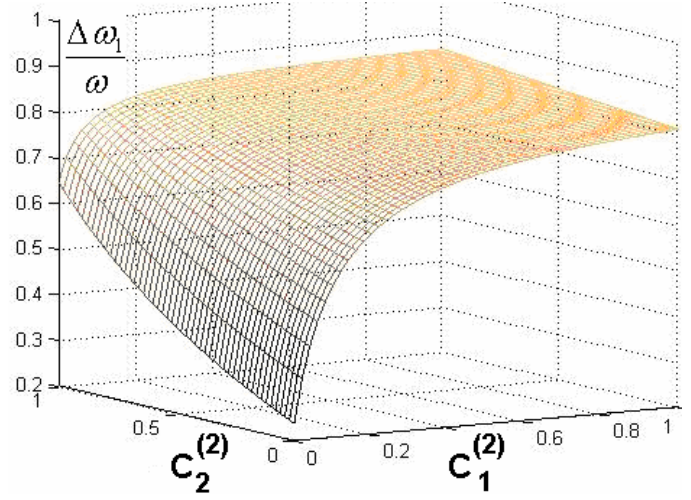

b

Fig. 2. Relative width of the lowest bandgap $\Delta \omega_{1} / \omega$ of the composite superlattice comprising the alternating silicon and liquid-crystal layers vs. concentrations of the admixture layers $\left(a_{1} / a_{2}=1\right)$. Surface (a) refers the case of $\varepsilon_{1}^{(2)} / \varepsilon_{1}^{(1)}=0.1$ and $\varepsilon_{2}^{(2)} / \varepsilon_{2}^{(1)}=0.2$ and surface (b) the case of $\varepsilon_{1}^{(2)} / \varepsilon_{1}^{(1)}=20$ and $\varepsilon_{2}^{(2)} / \varepsilon_{2}^{(1)}=0.2$.

In Fig. 2 the width of the lowest energy gap is plotted versus the concentrations $C_{1}^{(2)}$, $C_{2}^{(2)}$ of the admixture layers for the superlattice with alternating silicon and liquid-crystal layers. The energy gap $\Delta \omega_{1}$ vanishes at $\varepsilon_{1}^{(1)} f_{1}=\varepsilon_{2}^{(1)} f_{2}$ for the case (a) depicted in Fig. 2. 


\section{Conclusion}

Our results show that the optical characteristics of imperfect superlattices may be significantly altered owing to transformation of their polariton spectrum resulted from a presence of admixture layers. The theory developed is a basis for phenomenological description of a wide class of optical processes in non-ideal multilayered systems. Eqs. (2)-(5) and (7) allow numerical calculation of the concentration dependence of relevant optical characteristics. The essential quantities governing the propagation of electromagnetic waves through the media under consideration are the refractive indices, the photon gap width and the quantities defined by them, which can be measured directly (e.g., the light transmission coefficient). Graphic representation of the dependences $n_{ \pm}, \Delta \omega / \omega\left(C_{1}^{(2)}, C_{2}^{(2)}\right)$ (see Fig. 1 and 2) proves that character of the concentration dependence for the binary systems considered above differs for different concentration intervals. The case of non-ideal multilayered systems with a larger number of sublattices and components of alien layers (see [5]) supposes even a wider variety of specific behaviours of the refractive index and the gap width. This circumstance extends considerably the promises of modelling composite materials with predetermined properties.

\section{References}

1. Lourtioz J.-M., Benisty H., Berger V., Gerard J.-M., Maystre D. and Tchelnokov A. Photonic Crystals: Towards Nanoscale Photonic Devices. New York: Springer (2005).

2. Belotelov V I, Kotov V A, Zvezdin A K, Alameh K and Vasiliev M V. Optical properties of the magnetic crystals at the oblique light incidence. V. Vernadsky Taurida National University. Proc. of International Conference "Functional Materials" (2005) p. 132.

3. Lyubchanskii I L, Dadoenkova N N, Lyubchanskii M L, Shapovalov E A, Lakhtakia A and Rasing Th, 2004. One-dimensional bigyrotropic magnetic photonic crystals. Appl. Phys. Lett. 85: 5932-5934.

4. Nau D, Schonhardt A, Chigrin D N, Kroha H, Christ A and Giessen H, 2007. Polariton bandstructure of disordered metallic photonic crystal slabs. Phys. Stat. Solidi (b). 244: $1262-1269$.

5. Rumyantsev V V and Fedorov S A, 2007. Polariton spectrum of imperfect lyotropic lamellar system. Liquid Crystals and Their Application. 1(19): 67-74.

6. Pokatilov E.P., Fomin V.M. and Beril S.I. Vibrational Excitations, Polarons, and Excitons in Multilayer Systems and Superlattices. Chisinau: Shtiintsa (1990).

7. Yariv A. and Yeh P. Optical waves in crystals. New York: John Willey \& Sons, Inc. (1987).

8. Lyubchanskii I L, Dadoenkova N N, Lyubchanskii M L, Shapovalov E A, Lee Y P and Rasing Th. Light propagation in magnetic photinic crystals: oblique incidence. V. Vernadsky Taurida National University. International Conference "Functional Mate- 
rials". Proceedings (2005) p. 133.

9. Parmenter R H, 1955. Energy Levels of a Disordered Alloy. Phys. Rev. 97: 587-698.

10. Dargan T G, Capaz R B and Koiler Belita, 1997. Critical Analysis of the Virtual Crystal Approximation. Brazilian J. Phys. 27/A: 299-304.

11. Ziman J.M. Models of disorder. The theoretical physics of homogeneously disordered systems. New York: John Willey \& Sons, Inc. (1979).

12. Mezrag F, Aouina N Y and Bouarissa N, 2006. Optoelectronic and dielectric properties of $\mathrm{GaAs}_{x} \mathrm{Sb}_{1-x}$ ternary alloys. J. Mater. Sci. 41: 5323-5328.

13. Los' V F, 1987. Projection operator method in the theory of disordered systems. 1. Spectra of quasiparticles. Theor. and Math. Phys. 73: 85-102. 